\title{
Comparative Vibrational Spectra of Pilosine and Epiisopilosine crystals
}

\author{
R.R.F. Bento, ${ }^{*}$ L.E. da Silva, and J.L.B. Faria \\ Universidade Federal do Mato Grosso, Cuiabá-MT, Brazil \\ P.T.C. Freire, M.C.F. de Oliveira, and N.R. Romero \\ Universidade Federal do Ceará, Fortaleza-CE, Brazil \\ A.M.R. Teixeira \\ Universidade Estadual Vale do Acaraú, Sobral-CE, Brazil \\ F.M. Pontes \\ Universidade Estadual Paulista, Bauru-SP, Brasil
}

(Received on 2 February, 2010)

\begin{abstract}
This paper describes the study of two alkaloids extracted from Pilocarpus Microphyllus (Rutaceae): pilosine and epiisopilosine. These substances have potential application in the treatment of several diseases. In this work FT-Raman and the FT-IR spectra of pilosine and epiisopilosine were investigated at $300 \mathrm{~K}$. Vibrational wavenumber and wave vector have been predicted using density functional theory (B3LYP) calculations with the 6-31 G(d,p) basis set. A comparison with experiment, allowed us to assign most of the normal modes of the crystals.
\end{abstract}

Keywords: Raman scattering; infrared spectroscopy; normal modes; pilosine; epiisopilosine

\section{INTRODUCTION}

Pilocarpus Microphyllus (Rutaceae) is originated from the Amazon region in Brazil where it is known as jaborandi [1]. This plant is a rich source of imidazole alkaloids, of which pilosine and epiisopilosine are the best known and the only substances that are economically exploited. Pilosine and epiisopilosine (an isomer of pilosine), have been well studied and their biological properties explored not only for the treatment of glaucoma [2] but also as a stimulant of sweat and lachrymal glands [3]. These alkaloids are also a pilocarpinelike peripheric stimulant of parasympatic nervous system, although in higher dosis [4]. The possibility of production of pilosine and epiisopilosine by callus cell lines in bioreactors has been evaluated, with the objective of protecting the jaborandi plant from uncontrolled exploitation [5].

Pilosine has the molecular formula $\mathrm{C}_{16} \mathrm{H}_{18} \mathrm{O}_{3} \mathrm{~N}_{2}$ and is a monoacidic base which contains one $\mathrm{N}$-methyl group [6]. The presence of a lactonic core is achieved by titration with hot alkali solution. The alkaloid also contains an alcoholic hydroxyl group, which can be confirmed by the preparation of an amorphous benzoyl derivative, and by the dehydration reaction of the alkaloid with hot acetic anhydride to anhydropilosine, $\mathrm{C}_{16} \mathrm{H}_{16} \mathrm{O}_{2} \mathrm{~N}_{2}$ [7].

In this work an infrared analysis and a Raman scattering study in the spectral range $40 \mathrm{~cm}^{-1}$ to $4000 \mathrm{~cm}^{-1}$ of pilosine and epiisopilosine crystals obtained from Pilocarpus jaborandi, is reported, in order to assign the normal modes of vibrations of the material. In addition a Density Functional Theory (DFT) calculation was performed.

\footnotetext{
*Electronic address: ricardobento@fisica.ufmt.br
}

\section{EXPERIMENTAL}

FT-Raman spectrum was taken using a Bruker RFS100/S FTR system and a D418-T detector, with the sample excited by means of the $1064 \mathrm{~nm}$ line of a Nd:YAG laser. Infrared spectrum was obtained by using an Equinox/55 (Bruker) Fourier Transformed Infrared (FTIR) spectrometer. FT-Raman and FT-IR spectra were collected from samples confined in screw cap standard chromatographic glass vials, at a nominal resolution of $4 \mathrm{~cm}^{-1}$ accumulating 60 scans per spectra and using a laser power of $150 \mathrm{~mW}$. In order to record IR spectra we have grinded the sample in an agate mortar to minimize scattering on the particle surface and prepared a pellet with $\mathrm{KBr}$ by mixing it with the sample until a uniform mixture was obtained. On the other hand, in order to record the Raman spectra we have used slightly compacted powder of the sample in a specific sample holder.

\section{COMPUTATIONAL METHOD}

Density functional theory (DFT) calculations were carried out using the Gaussian 98 programme package [8]. The B3LYP functional was used with the 6-31 G(d,p) basis set. The calculations were performed using an isolated molecule of pilosine and epiisopilosine. This structure was optimized and the vibrational wavenumbers were then calculated. The output file contained the optimized structure, the vibrational frequencies in the harmonic approximation, and the atomic displacements for each mode. At the optimized structure of the molecule, no imaginary frequency was obtained, proving that a true minimum of the potential energy surface was found. The calculated vibrational wavenumbers are compared with experimental Raman and IR frequencies. 


\section{RESULTS AND DISCUSSION}

Figure 1 shows the molecular structure of the pilosine. This labelling will be used in describing the parameters for optimized structure and the molecular wave vectors. The molecular structure of the epiisopilosine is shown in Figure 2.

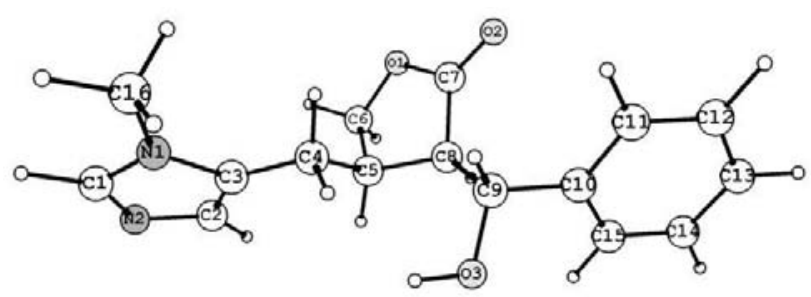

FIG. 1: The molecular structure of an isolated molecule of pilosine $\mathrm{C}_{16} \mathrm{H}_{18} \mathrm{O}_{3} \mathrm{~N}_{2}$.

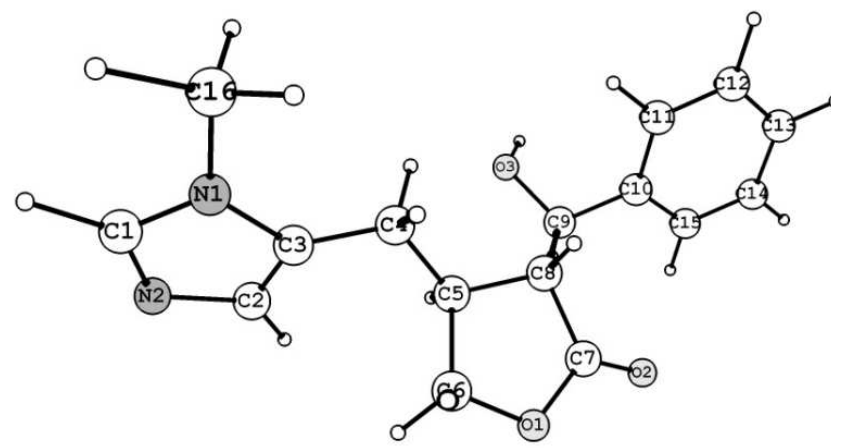

FIG. 2: The molecular structure of an isolated molecule of epiisopilosine $\mathrm{C}_{16} \mathrm{H}_{18} \mathrm{O}_{3} \mathrm{~N}_{2}$.

Comparative FT-Raman spectrum and FT-infrared (FT-IR) spectrum of pilosine and epiisopilosine are presented respectively, in Figures 3 and 4.

Each one of the isomers pilosine and epiisopilosine have 39 atoms in its molecular structure, therefore, we have 111 molecular vibrations in each one of molecules.

The Table I lists a detailed description of assignments for vibrational wavenumbers of pilosine and epiisopilosine crystals (unhappyly, up to now, the crystal structure of the two materials is not available). In the first column the calculated values for the wavenumbers are given. We also present the experimental wavenumber values for the crystal obtained by FT-Raman and FT-IR spectroscopies (the second and third columns, respectively). The values placed without parenthesis correspond to the frequencies associated to the pilosine molecule and the values placed within parenthesis correspond to the frequencies associated the epiisopilosine molecule. The fourth and in the last column we give the assignment of the bands.
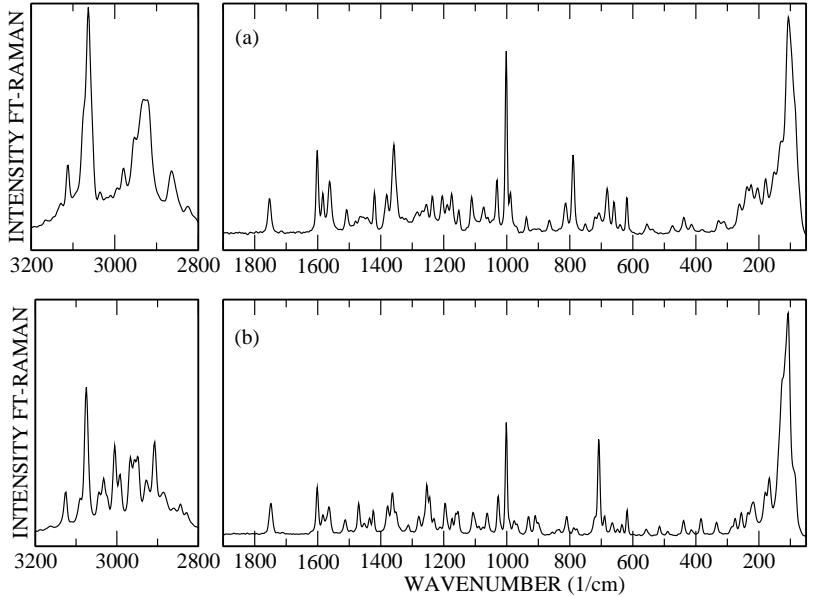

FIG. 3: FT-Raman spectrum of crystals of (a) pilosine and (b) epiisopilosine.
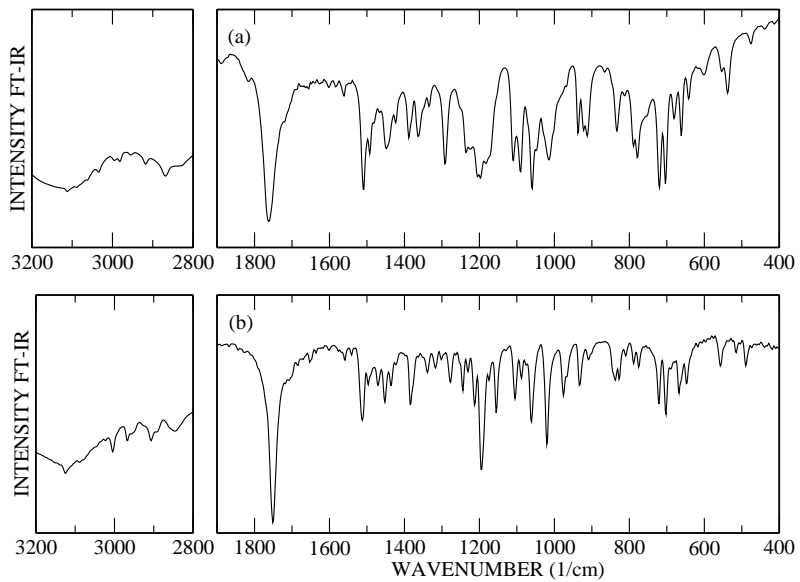

FIG. 4: FT-IR spectrum of crystals of (a) pilosine and (b) epiisopilosine.

The nomenclature used in the assignments of molecular vibrations are as follows: $\mathrm{r}=$ rocking; $\tau=$ twisting; $\mathrm{sc}=$ scissoring; wag $=$ wagging; $\delta=$ deformation; $\delta_{i p}=$ deformation in plane; $\delta_{o o p}=$ deformation out of plane; $v_{s}=$ symmetric stretching and $v_{a s}=$ asymmetric stretching. To better classify the molecular vibrations, we refer to the three rings in the table of assignments of vibrational modes as follows: R1 ring that contains a functional group type-benzene, R2 ring that contains a functional group type- $\gamma$-butyrolactone and R3 ring that contains a functional group type-1-methylimidazole.

The imidazole ring is present in several substances of biological interest, as for example, L-histidine amino acid,[9$11]$ and in other substances [12, 13]. Their vibrations spread over a large spectral range of wavenumbers. Lactone, the other ring, is also found in several different substances [1416]; for some of them spectroscopic studies have revealed the wavenumber of the main vibrations [15].

The assignment for pilosine and epiisopilosine shows that most of the bands observed through FT-Raman and FT-IR 
spectroscopies correspond to a mixture of vibrational modes. The mixture of modes is common in molecules of $\mathrm{C} 1$ site symmetry. The superposition of modes precludes a direct identification of the bands. However, an effort was carried out through this work to make a detailed description of assignments of vibrational modes of the crystals.

Calculations show that at low wavenumber $\left(\omega_{\text {calc }}<674\right.$ $\mathrm{cm}^{-1}$ ) the whole structure presents deformation vibrations $\delta$ (all structure). In this region it is expected to be observed bands associated to the lattice vibrations, some internal modes are also present. For example, torsional vibrations of the two rings are observed together with lattice modes at very low wavenumber. This should be expected because the rings are very large structures; so, we assign the bands in this spectral region as a mixture of lattice modes and torsional vibrations of the rings.

TABLE I: Assignment of FT-Raman and FT-IR wavenumber of Isomers Pilosine and Epiisopilosine crystals. The wavenumbers for epiisopilosine are in parentheses.

\begin{tabular}{|c|c|c|c|c|}
\hline$\omega_{\text {calc }}$ & $\omega_{F T-\text { Ramam }}$ & $\omega_{F T-I R}$ & Vibrational Modes of Pilosine & Vibrational Modes of Epiisopilosine \\
\hline $25(20)$ & & & $\delta($ all structure $)$ & $\delta($ all structure $)$ \\
\hline $26(27)$ & & & $\delta$ (all structure) & $\delta$ (all structure) \\
\hline $40(38)$ & & & $\delta($ all structure $)$ & $\delta($ all structure) \\
\hline $48(54)$ & & & $\delta($ all structure $)$ & $\delta($ all structure $)$ \\
\hline $78(58)$ & & & $\delta$ (all structure) & $\delta$ (all structure) \\
\hline $83(84)$ & $91(83)$ & & $\delta($ all structure $)$ & $\delta($ all structure $)$ \\
\hline $112(103)$ & $108(105)$ & & $\delta($ all structure $)$ & $\delta($ all structure $)$ \\
\hline $116(124)$ & $130(128)$ & & $\mathrm{r}\left(\mathrm{C} 16 \mathrm{H}_{3}\right)$ & $\delta($ all structure) \\
\hline $139(150)$ & $152(167)$ & & $\delta$ (all structure) & $\delta$ (all structure) \\
\hline $182(175)$ & $179(180)$ & & $\delta$ (all structure) & $\delta_{\text {oop }}(\mathrm{R} 2) ; \mathrm{r}\left(\mathrm{C} 4 \mathrm{H}_{2}, \mathrm{C} \mathrm{H}_{2}\right)$ \\
\hline $190(206)$ & & & $\delta($ all structure $)$ & $\delta_{\text {oop }}(\mathrm{R} 2, \mathrm{R} 3) ; \mathrm{r}\left(\mathrm{C} 16 \mathrm{H}_{3}, \mathrm{C} 4 \mathrm{H}_{2}, \mathrm{C} 9 \mathrm{O} 3 \mathrm{H}\right)$ \\
\hline $208(210)$ & 204 & & $\delta($ all structure $)$ & $\delta($ all structure $)$ \\
\hline $225(216)$ & $224(222)$ & & $\delta($ all structure $)$ & $\delta($ all structure $)$ \\
\hline $243(226)$ & $238(234)$ & & $\delta($ all structure $)$ & $\delta($ all structure $)$ \\
\hline $253(250)$ & $(256)$ & & $\delta($ all structure $)$ & $\delta($ all structure $)$ \\
\hline $266(284)$ & $260(272)$ & & $\delta(\mathrm{O} 3 \mathrm{H}) ; \mathrm{r}\left(\mathrm{C} 4 \mathrm{H}_{2}, \mathrm{C}_{16 \mathrm{H}_{3}}\right)$ & $\delta_{\text {oop }}(\mathrm{R} 2, \mathrm{R} 3) ; \delta(\mathrm{O} 3 \mathrm{H}) ; \mathrm{r}\left(\mathrm{C} 4 \mathrm{H}_{2}, \mathrm{C}_{16 \mathrm{H}_{3}}\right)$ \\
\hline $279(314)$ & $(281)$ & & $\delta_{o o p}(\mathrm{R} 1) ; \delta(\mathrm{O} 3 \mathrm{H}) ; \mathrm{r}\left(\mathrm{C}_{4} \mathrm{H}_{2}\right)$ & $\begin{array}{l}\text { sc(C8C9C10,C8C5C4,C5C4C3); } \\
\delta_{\text {oop }}(\mathrm{R} 1) ; \delta(\mathrm{O} 3 \mathrm{H}) ; \mathrm{r}\left(\mathrm{C} 4 \mathrm{H}_{2}\right)\end{array}$ \\
\hline $317(330)$ & $315(334)$ & & $\mathrm{r}(\mathrm{C} 9 \mathrm{O} 3 \mathrm{H})$ & $\delta(\mathrm{O} 3 \mathrm{H})$ \\
\hline $385(352)$ & $(384)$ & & $\delta($ all structure $)$ & $\delta(\mathrm{O} 3 \mathrm{H})$ \\
\hline $390(409)$ & 413 & 413 & $\delta($ all structure $)$ & $\delta($ all structure) \\
\hline $416(418)$ & $438(414)$ & 438 & $\delta_{o o p}(\mathrm{R} 1)$ & $\delta_{o o p}(\mathrm{R} 1)$ \\
\hline $454(426)$ & $447(439)$ & 458 & $\delta$ (all structure) & $\delta$ (all structure) \\
\hline $489(496)$ & 474 & $476(490)$ & $\delta_{o o p}(\mathrm{R} 2) ; \delta_{i p}(\mathrm{R} 3)$ & $\delta_{\text {oop }}(\mathrm{R} 2) ; \delta_{i p}(\mathrm{R} 3) ; \mathrm{sc}(\mathrm{C} 1 \mathrm{~N} 1 \mathrm{C} 16)$ \\
\hline $520(523)$ & $538(516)$ & $537(516)$ & $\delta_{o o p}(\mathrm{R} 1) ; \delta_{i p}(\mathrm{R} 2)$ & $\delta_{\text {oop }}(\mathrm{R} 1, \mathrm{R} 2)$ \\
\hline $586(579)$ & $557(558)$ & $545(557)$ & $\delta_{o o p}(\mathrm{R} 2)$ & $\delta_{\text {oop }}(\mathrm{R} 2)$ \\
\hline $615(614)$ & $619(618)$ & 599 & $\delta_{\text {oop }}(\mathrm{R} 1) ; \delta_{\text {oop }}(\mathrm{R} 2)$ & $\delta($ all structure $)$ \\
\hline $631(632)$ & & 625 & $\delta_{o o p}(\mathrm{R} 3)$ & $\delta_{i p}(\mathrm{R} 1) ; \delta_{o o p}(\mathrm{R} 2, \mathrm{R} 3)$ \\
\hline $633(633)$ & $(634)$ & & $\delta_{i p}(\mathrm{R} 1) ; \mathrm{wag}(\mathrm{C} 9 \mathrm{O} 3 \mathrm{H})$ & $\delta_{i p}(\mathrm{R} 1) ; \mathrm{sc}(\mathrm{C} 8 \mathrm{C} 9 \mathrm{C} 10), \delta_{o o p}(\mathrm{R} 3)$ \\
\hline $636(637)$ & 640 & 642 & $\delta_{i p}(\mathrm{R} 1) ; \delta(\mathrm{C} 8 \mathrm{H})$ & 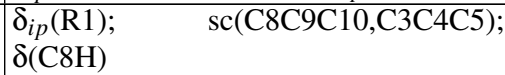 \\
\hline $659(659)$ & 659 (649) & $661(647)$ & $\begin{array}{lll}\delta_{i p}(\mathrm{R} 3) ; & v_{S}(\mathrm{C} 16 \mathrm{~N} 1) ; & \delta_{o o p}(\mathrm{R} 2) ; \\
\operatorname{wag}\left(\mathrm{C} 4 \mathrm{H}_{2}\right) & & \\
\end{array}$ & $\begin{array}{ll}\delta_{i p}(\mathrm{R} 3) ; \quad \delta_{o o p}(\mathrm{R} 2) ; & v \mathrm{~s}(\mathrm{C} 16 \mathrm{~N} 1) \\
\mathrm{sc}(\mathrm{C} 8 \mathrm{C} 9 \mathrm{C} 10)\end{array}$ \\
\hline $674(674)$ & $(665)$ & $(666)$ & $\delta_{\text {oop }}(\mathrm{R} 3)$ & $\delta_{\text {oop }}(\mathrm{R} 2, \mathrm{R} 3)$ \\
\hline $710(676)$ & $681(689)$ & 680 & $\delta_{o o p}(\mathrm{C} 11 \mathrm{H}, \mathrm{C} 12 \mathrm{H}, \mathrm{C} 13 \mathrm{H}, \mathrm{C} 14 \mathrm{H}, \mathrm{C} 15 \mathrm{H})$ & $\delta_{\text {oop }}(\mathrm{R} 2, \mathrm{R} 3)$ \\
\hline $712(712)$ & $708(708)$ & $703(703)$ & $\begin{array}{l}\delta_{o o p}(\mathrm{C} 11 \mathrm{H}, \mathrm{C} 12 \mathrm{H}, \mathrm{C} 13 \mathrm{H}, \mathrm{C} 14 \mathrm{H}, \mathrm{C} 15 \mathrm{H}) \\
\delta_{i p}(\mathrm{R} 2) ; \mathrm{r}\left(\mathrm{C} 4 \mathrm{H}_{2}\right)\end{array}$ & $\begin{array}{l}\delta_{o o p}(\mathrm{C} 11 \mathrm{H}, \mathrm{C} 12 \mathrm{H}, \mathrm{C} 13 \mathrm{H}, \mathrm{C} 14 \mathrm{H}, \mathrm{C} 15 \mathrm{H}) \\
\delta_{\text {oop }}(\mathrm{R} 2)\end{array}$ \\
\hline $730(714)$ & 721 & $720(722)$ & $\begin{array}{lll}\delta_{i p}(\mathrm{R} 3) ; & v_{S}(\mathrm{C} 16 \mathrm{~N} 1) ; & \delta_{o o p}(\mathrm{R} 2) \\
\mathrm{r}\left(\mathrm{C} 4 \mathrm{H}_{2}\right) & \end{array}$ & $\delta_{o o p}(\mathrm{C} 11 \mathrm{H}, \mathrm{C} 12 \mathrm{H}, \mathrm{C} 13 \mathrm{H}, \mathrm{C} 14 \mathrm{H}, \mathrm{C} 15 \mathrm{H})$ \\
\hline $775(769)$ & $751(780)$ & $754(775)$ & $\delta_{o o p}(\mathrm{C} 11 \mathrm{H}, \mathrm{C} 12 \mathrm{H}, \mathrm{C} 13 \mathrm{H}, \mathrm{C} 14 \mathrm{H}, \mathrm{C} 15 \mathrm{H})$ & $\begin{array}{l}\delta_{o o p}(\mathrm{C} 12 \mathrm{H}, \mathrm{C} 13 \mathrm{H}, \mathrm{C} 14 \mathrm{H}, \mathrm{C} 15 \mathrm{H}, \mathrm{C} 16 \mathrm{H}) \\
\delta_{i p}(\mathrm{R} 3) ; \mathrm{v}(\mathrm{C} 16 \mathrm{~N} 1) ; \mathrm{sc}(\mathrm{C} 3 \mathrm{C} 4 \mathrm{C} 5)\end{array}$ \\
\hline $798(784)$ & $789(788)$ & $784(788)$ & $\delta_{i p}(\mathrm{R} 2, \mathrm{R} 3) ; v_{s}(\mathrm{C} 16 \mathrm{~N} 1) ; \delta_{o o p}(\mathrm{C} 1 \mathrm{H})$ & $\begin{array}{l}\delta_{\text {oop }}(\mathrm{C} 11 \mathrm{H}, \mathrm{C} 12 \mathrm{H}, \mathrm{C} 13 \mathrm{H}, \mathrm{C} 14 \mathrm{H} \\
\mathrm{C} 15 \mathrm{H}) ; \quad \delta_{i p}(\mathrm{R} 3) ; \quad v(\mathrm{C} 16 \mathrm{~N} 1, \mathrm{C} 3 \mathrm{C} 4) \\
\mathrm{sc}(\mathrm{C} 8 \mathrm{C} 9 \mathrm{C} 10, \mathrm{C} 3 \mathrm{C} 4 \mathrm{C} 5)\end{array}$ \\
\hline $803(795)$ & $813(810)$ & $814(810)$ & $\delta_{\text {oop }}(\mathrm{C} 1 \mathrm{H})$ & $\delta_{\text {oop }}(\mathrm{C} 1 \mathrm{H})$ \\
\hline $834(834)$ & & 833 & $\delta_{o o p}(\mathrm{C} 2 \mathrm{H})$ & $\begin{array}{lll}\delta_{o o p}(\mathrm{C} 2 \mathrm{H}) ; & \delta_{i p}(\mathrm{R} 2) ; & \delta(\mathrm{O} 3 \mathrm{H}) \\
\mathrm{v}_{s}(\mathrm{C} 9 \mathrm{C} 10)\end{array}$ \\
\hline
\end{tabular}


TABLE I - continued from previous page

\begin{tabular}{|c|c|c|c|c|}
\hline$\omega_{\text {calc }}$ & $\omega_{F T-\text { Ramam }}$ & $\omega_{F T-I R}$ & Vibrational Modes of Pilosine & Vibrational Modes of Epiisopilosine \\
\hline $840(837)$ & $(837)$ & $(839)$ & $\begin{array}{lll}\delta_{\text {oop }}(\mathrm{C} 2 \mathrm{H}) ; & \delta_{i p}(\mathrm{R} 2) ; & \delta(\mathrm{O} 3 \mathrm{H}) \\
v_{s}(\mathrm{C} 9 \mathrm{C} 10)\end{array}$ & $\delta_{o o p}(\mathrm{C} 2 \mathrm{H}) ; \delta(\mathrm{O} 3 \mathrm{H}) ; v_{s}(\mathrm{C} 9 \mathrm{C} 10)$ \\
\hline $857(861)$ & $(855)$ & & $\delta_{\text {oop }}(\mathrm{C} 11 \mathrm{H}, \mathrm{C} 12, \mathrm{C} 14 \mathrm{H}, \mathrm{C} 15 \mathrm{H})$ & $\delta_{\text {oop }}(\mathrm{C} 11 \mathrm{H}, \mathrm{C} 12 \mathrm{H}, \mathrm{C} 14 \mathrm{H}, \mathrm{C} 15 \mathrm{H})$ \\
\hline $865(889)$ & 865 & 867 & $\begin{array}{l}\delta_{o o p}(\mathrm{C} 11 \mathrm{H}, \mathrm{C} 13 \mathrm{H}, \mathrm{C} 14 \mathrm{H}, \mathrm{C} 15 \mathrm{H}) \\
\delta_{i p}(\mathrm{R} 2) ; \mathrm{v}_{s}(\mathrm{C} 4 \mathrm{C} 5)\end{array}$ & $\delta_{\text {oop }}(\mathrm{C} 2 \mathrm{H}) ; \mathrm{r}(\mathrm{C} 4 \mathrm{H} 2) ; \delta_{i p}(\mathrm{R} 2)$ \\
\hline $898(911)$ & $899(908)$ & $(911)$ & $\begin{array}{l}\delta_{\text {oop }}(\mathrm{C} 11 \mathrm{H}, \mathrm{C} 12 \mathrm{H}, \mathrm{C} 13 \mathrm{H}, \mathrm{C} 15 \mathrm{H}) ; \\
\mathrm{r}\left(\mathrm{C} 4 \mathrm{H}_{2}\right) ; \delta_{i p}(\mathrm{R} 2)\end{array}$ & $\delta_{\text {oop }}(\mathrm{R} 2) ; \delta_{i p}(\mathrm{R} 3) ; \mathrm{r}\left(\mathrm{C} 6 \mathrm{H}_{2}\right)$ \\
\hline $925(920)$ & 914 & $911(933)$ & $\begin{array}{l}\delta_{\text {oop }}(\mathrm{C} 11 \mathrm{H}, \mathrm{C} 12 \mathrm{H}, \mathrm{C} 13 \mathrm{H}, \mathrm{C} 15 \mathrm{H}) \\
\mathrm{r}\left(\mathrm{C} 4 \mathrm{H}_{2}, \mathrm{C} 6 \mathrm{H}_{2}\right) ; \delta(\mathrm{C} 5 \mathrm{H}, \mathrm{C} 8 \mathrm{H})\end{array}$ & $\begin{array}{l}\delta_{o o p}(\mathrm{C} 11 \mathrm{H}, \mathrm{C} 13 \mathrm{H}, \mathrm{C} 14 \mathrm{H}, \mathrm{C} 15 \mathrm{H}) \\
\mathrm{r}\left(\mathrm{C} 2 \mathrm{H}_{2}, \mathrm{C} 4 \mathrm{H}_{2}\right) ; \delta(\mathrm{C} 5 \mathrm{H}, \mathrm{C} 8 \mathrm{H})\end{array}$ \\
\hline $939(936)$ & $938(931)$ & 937 & $\begin{array}{ll}\delta_{i p}(\mathrm{R} 3) ; & \mathrm{r}\left(\mathrm{C} 4 \mathrm{H}_{2}, \mathrm{C}_{6} \mathrm{H}_{2}, \mathrm{C}_{16 \mathrm{H}}\right) \\
\delta(\mathrm{C} 5 \mathrm{H}) & \end{array}$ & $\begin{array}{l}\delta_{o o p}(\mathrm{C} 11 \mathrm{H}, \mathrm{C} 13 \mathrm{H}, \mathrm{C} 15 \mathrm{H}) ; \quad \delta_{i p} \\
\mathrm{r}\left(\mathrm{C} 6 \mathrm{H}_{2}\right) ; \delta(\mathrm{C} 1 \mathrm{H}, \mathrm{C} 2 \mathrm{H}, \mathrm{C} 5 \mathrm{H})\end{array}$ \\
\hline $942(940)$ & 971 & $(966)$ & $\begin{array}{l}\left.\delta_{\text {oop }}(\mathrm{C} 11 \mathrm{H}), \mathrm{C} 12 \mathrm{H}, \mathrm{C} 13 \mathrm{H}, \mathrm{C} 14 \mathrm{H}, \mathrm{C} 15 \mathrm{H}\right) \\
\delta_{i p}(\mathrm{R} 2) ; \mathrm{r}\left(\mathrm{C} 4 \mathrm{H}_{2}, \mathrm{C} 6 \mathrm{H}_{2}\right)\end{array}$ & $\begin{array}{l}\delta_{o o p}(\mathrm{C} 11 \mathrm{H}, \mathrm{C} 13 \mathrm{H}, \mathrm{C} 14 \mathrm{H}, \mathrm{C} 15 \mathrm{H}) \\
\delta_{i p}(\mathrm{R} 3) ; \mathrm{r}\left(\mathrm{C} 8 \mathrm{H}_{2}, \mathrm{C} 6 \mathrm{H}_{2}, \mathrm{C} 16 \mathrm{H}_{3}\right) \\
\end{array}$ \\
\hline $973(976)$ & $986(974)$ & $(976)$ & $\delta_{\text {oop }}(\mathrm{C} 11 \mathrm{H}, \mathrm{C} 12 \mathrm{H}, \mathrm{C} 13 \mathrm{H}, \mathrm{C} 14 \mathrm{H}, \mathrm{C} 15 \mathrm{H})$ & $\delta_{o o p}(\mathrm{C} 11 \mathrm{H}, \mathrm{C} 12 \mathrm{H}, \mathrm{C} 14 \mathrm{H}, \mathrm{C} 15 \mathrm{H})$ \\
\hline $982(999)$ & $1001(1001)$ & $(1020)$ & $\delta_{i p}(\mathrm{R} 2) ; \mathrm{r}\left(\mathrm{C} 4 \mathrm{H}_{2}\right)$ & $\delta_{\text {oop }}(\mathrm{C} 11 \mathrm{H}, \mathrm{C} 12 \mathrm{H}, \mathrm{C} 13 \mathrm{H}, \mathrm{C} 14 \mathrm{H}, \mathrm{C} 15 \mathrm{H})$ \\
\hline $997(1017)$ & & & $\delta_{\text {oop }}(\mathrm{C} 11 \mathrm{H}, \mathrm{C} 12 \mathrm{H}, \mathrm{C} 13 \mathrm{H}, \mathrm{C} 14 \mathrm{H}, \mathrm{C} 15 \mathrm{H})$ & $\delta_{i p}(\mathrm{R} 1)$ \\
\hline $1014(1032)$ & $(1028)$ & 1014 & $v_{s}(\mathrm{C} 7 \mathrm{O} 1, \mathrm{C} 4 \mathrm{C} 5) ; \mathrm{r}\left(\mathrm{C} 6 \mathrm{H}_{2}\right) ; \delta(\mathrm{C} 5 \mathrm{H})$ & $\begin{array}{lll}v_{s}(\mathrm{C} 9 \mathrm{C} 10) ; & \delta(\mathrm{O} 3 \mathrm{H}) ; & \mathrm{r}\left(\mathrm{C}_{4} \mathrm{H}_{2}\right) \\
\delta_{\text {oop }}(\mathrm{R} 2) & & \\
\end{array}$ \\
\hline $1019(1055)$ & 1031 & & $\delta_{i p}(\mathrm{R} 1)$ & $\delta_{i p}(\mathrm{R} 1)$ \\
\hline $1054(1066)$ & 1045 & 1047 & $\delta_{i p}(\mathrm{R} 1)$ & $\begin{array}{l}\delta_{i p}(\mathrm{C} 11 \mathrm{H}, \mathrm{C} 13 \mathrm{H}, \mathrm{C} 14 \mathrm{H}) ; \\
v_{s}(\mathrm{C} 6 \mathrm{O} 1) ; \delta(\mathrm{O} 3 \mathrm{H})\end{array}$ \\
\hline $1062(1069)$ & $1059(1062)$ & $1061(1061)$ & $\begin{array}{l}\delta_{i p}(\mathrm{C} 11 \mathrm{H}, \mathrm{C} 12 \mathrm{H}, \mathrm{C} 13 \mathrm{H}, \mathrm{C} 14 \mathrm{H}, \mathrm{C} 15 \mathrm{H}) \\
v_{s}(\mathrm{C} 9 \mathrm{O} 3, \mathrm{C} 5 \mathrm{C} 6, \mathrm{C} 7 \mathrm{O} 1) \\
\end{array}$ & $\begin{array}{l}\delta_{i p}(\mathrm{C} 11 \mathrm{H}, \mathrm{C} 12 \mathrm{H}, \mathrm{C} 13 \mathrm{H}, \mathrm{C} 14 \mathrm{H}, \mathrm{C} 15 \mathrm{H}) \\
\mathrm{v}_{s}(\mathrm{C} 9 \mathrm{O} 3, \mathrm{C} 5 \mathrm{C} 6) ; \delta_{i p}(\mathrm{R} 2)\end{array}$ \\
\hline $1074(1075)$ & $1074(1081)$ & $(1076)$ & $\begin{array}{l}\mathrm{r}\left(\mathrm{C} 4 \mathrm{H}_{2}, \mathrm{C}_{6} \mathrm{H}_{2}, \mathrm{C} 16 \mathrm{H}_{3}\right) \\
\mathrm{v}_{S}(\mathrm{C} 5 \mathrm{C} 6, \mathrm{C} 7 \mathrm{O} 1, \mathrm{C} 1 \mathrm{~N} 1) \\
\end{array}$ & $\delta_{i p}(\mathrm{R} 3) ; v_{s}(\mathrm{C} 1 \mathrm{~N} 1) ; \mathrm{r}\left(\mathrm{C} 16 \mathrm{H}_{3}\right)$ \\
\hline $1078(1092)$ & & $1091(1088)$ & $\mathrm{r}\left(\mathrm{C} 16 \mathrm{H}_{3}\right) ; v_{s}(\mathrm{C} 1 \mathrm{~N} 1)$ & \begin{tabular}{|lcl}
$\delta_{i p}(\mathrm{R} 2 ;$ & $\mathrm{R} 3) ;$ & $v_{s}(\mathrm{C} 3 \mathrm{~N} 1) ;$ \\
$\mathrm{v}_{s}(\mathrm{C} 3 \mathrm{C} 4, \mathrm{C} 5 \mathrm{C} 6) ; \mathrm{r}\left(\mathrm{C} 16 \mathrm{H}_{3}\right)$ & \\
\end{tabular} \\
\hline $1106(1103)$ & $(1106)$ & $(1105)$ & $\begin{array}{lcc}v_{s}(\mathrm{C} 9 \mathrm{O} 3) ; & v_{s}(\mathrm{C} 7 \mathrm{O} 1) ; & \mathrm{r}\left(\mathrm{C} 6 \mathrm{H}_{2}\right) \\
\tau\left(\mathrm{C} 4 \mathrm{H}_{2}\right) ; \delta(\mathrm{C} 5 \mathrm{H}) ; \delta(\mathrm{C} 8 \mathrm{H}) & \\
\end{array}$ & $\begin{array}{l}\delta_{i p}(\mathrm{R} 2) ; v_{s}(\mathrm{C} 5 \mathrm{C} 6, \mathrm{C} 9 \mathrm{O} 3) ; \mathrm{r}\left(\mathrm{C}_{\mathrm{H}} \mathrm{H}_{2}\right) ; \\
\delta(\mathrm{C} 5 \mathrm{H}, \mathrm{C} 8 \mathrm{H}) ; \delta(\mathrm{O} 3 \mathrm{H}) ; \tau\left(\mathrm{C} 4 \mathrm{H}_{2}\right)\end{array}$ \\
\hline $1112(1112)$ & 1112 & 1110 & $\begin{array}{lrr}\delta_{i p}(\mathrm{C} 11 \mathrm{H}) ; & \delta_{i p}(\mathrm{C} 12 \mathrm{H}) ; & \delta_{i p}(\mathrm{C} 12 \mathrm{H}) \\
\delta_{i p}(\mathrm{C} 14 \mathrm{H}) ; & \delta_{i p}(\mathrm{C} 15 \mathrm{H}) ; & \delta(\mathrm{O} 3 \mathrm{H}) \\
\delta(\mathrm{C} 5 \mathrm{H}) ; \delta(\mathrm{C} 8 \mathrm{H}) ; \delta(\mathrm{C} 9 \mathrm{H}) & \end{array}$ & $\begin{array}{l}\delta_{i p}(\mathrm{C} 11 \mathrm{H}, \mathrm{C} 12 \mathrm{H}, \mathrm{C} 13 \mathrm{H}, \mathrm{C} 14 \mathrm{H}, \mathrm{C} 15 \mathrm{H}) \\
\delta_{i p}(\mathrm{R} 2) ; \mathrm{v}_{s}(\mathrm{C} 5 \mathrm{C} 8) ; \operatorname{wag}\left(\mathrm{C} 4 \mathrm{H}_{2}\right)\end{array}$ \\
\hline $1114(1123)$ & & & $\begin{array}{l}\mathrm{r}\left(\mathrm{C} 6 \mathrm{H}_{2}\right) ; \tau\left(\mathrm{C}_{4} \mathrm{H}_{2}\right) ; v(\mathrm{C} 5 \mathrm{C} 8) ; \delta_{i p}(\mathrm{C} 1 \mathrm{H} ; \\
\mathrm{C} 2 \mathrm{H})\end{array}$ & $\begin{array}{l}\delta_{i p}(\mathrm{C} 11 \mathrm{H}, \mathrm{C} 12 \mathrm{H}, \mathrm{C} 13 \mathrm{H}, \mathrm{C} 14 \mathrm{H}, \mathrm{C} 15 \mathrm{H}) ; \\
\delta_{i p} \quad(\mathrm{R} 2) ; \quad v_{s}(\mathrm{C} 5 \mathrm{C} 8) ; \quad \operatorname{wag}\left(\mathrm{C}_{4} \mathrm{H}_{2}\right) ; \\
\delta(\mathrm{C} 2 \mathrm{H})\end{array}$ \\
\hline $1150(1150)$ & 1152 & & $\mathrm{r}\left(\mathrm{C} 16 \mathrm{H}_{3}\right)$ & $\mathrm{r}\left(16 \mathrm{H}_{3}\right)$ \\
\hline $1159(1157)$ & $(1158)$ & $(1155)$ & $\begin{array}{l}\mathrm{v}(\mathrm{C} 2 \mathrm{~N} 2) ; \\
\operatorname{wag}\left(\mathrm{C} 4 \mathrm{H}_{2}\right)\end{array}$ & $v(\mathrm{C} 2 \mathrm{~N} 2) ; \delta_{i p}(\mathrm{C} 1 \mathrm{H}, \mathrm{C} 2 \mathrm{H})$ \\
\hline $1165(1175)$ & $1174(1174)$ & & $\begin{array}{l}\delta_{i p}(\mathrm{R} 3) ; \\
v(\mathrm{C} 7 \mathrm{O} 1)\end{array} \quad \mathrm{r}\left(\mathrm{C}_{6} \mathrm{H}_{2}\right) ; \quad \delta(\mathrm{C} 5 \mathrm{H}, \mathrm{C} 8 \mathrm{H})$ & $\begin{array}{l}\delta_{i p}(\mathrm{R} 3) ; \quad \operatorname{wag}\left(\mathrm{C} 4 \mathrm{H}_{2} ; \mathrm{C}_{6} \mathrm{H}_{2}\right) \\
\delta(\mathrm{C} 5 \mathrm{H}, \mathrm{C} 8 \mathrm{H}, \mathrm{C} 9 \mathrm{H}) ; \mathrm{v}(\mathrm{C} 7 \mathrm{O} 1) ; \delta(\mathrm{O} 3 \mathrm{H})\end{array}$ \\
\hline $1179(1181)$ & & 1182 & $\begin{array}{l}\delta_{i p}(\mathrm{R} 2) ; \mathrm{r}\left(\mathrm{C} 6 \mathrm{H}_{2}, \mathrm{C} 16 \mathrm{H}_{3}\right) ; \operatorname{wag}\left(\mathrm{C}_{4} \mathrm{H}_{2}\right) \\
\delta(\mathrm{C} 5 \mathrm{H}, \mathrm{C} 8 \mathrm{H})\end{array}$ & $\begin{array}{l}\delta_{i p}(\mathrm{R} 3) ; \mathrm{r}\left(\mathrm{C} 6 \mathrm{H}_{2}, \mathrm{C}_{16} \mathrm{H}_{3}\right) ; \\
\delta(\mathrm{C} 5 \mathrm{H} ; \mathrm{C} 8 \mathrm{H})\end{array}$ \\
\hline $1186(1188)$ & 1189 & 1192 & $\begin{array}{l}\delta_{i p}(\mathrm{C} 11 \mathrm{H}) \\
\delta_{i p}(\mathrm{C} 12 \mathrm{H}, \mathrm{C} 13 \mathrm{H}, \mathrm{C} 14 \mathrm{H}, \mathrm{C} 15 \mathrm{H}) \\
v(\mathrm{C} 9 \mathrm{C} 10) ; \delta(\mathrm{O} 3 \mathrm{H})\end{array}$ & $\delta_{i p}(\mathrm{C} 11 \mathrm{H}, \mathrm{C} 12 \mathrm{H}, \mathrm{C} 13 \mathrm{H}, \mathrm{C} 14 \mathrm{H}, \mathrm{C} 15 \mathrm{H})$ \\
\hline $1191(1204)$ & $(1196)$ & $(1194)$ & $\begin{array}{l}\delta_{i p}(\mathrm{C} 11 \mathrm{H}, \mathrm{C} 12 \mathrm{H}, \mathrm{C} 13 \mathrm{H}, \mathrm{C} 14 \mathrm{H}, \mathrm{C} 15 \mathrm{H}) \\
v(\mathrm{C} 9 \mathrm{C} 10) ; \delta(\mathrm{O} 3 \mathrm{H})\end{array}$ & $\begin{array}{lr}\tau\left(\mathrm{C} 4 \mathrm{H}_{2}\right) ; & \delta_{i p}(\mathrm{C} 11 \mathrm{H}, \mathrm{C} 12 \mathrm{H}, \mathrm{C} 14 \mathrm{H}, \\
\mathrm{C} 15 \mathrm{H}) ; \delta(\mathrm{C} 5 \mathrm{H}, \mathrm{C} 8 \mathrm{H}, \mathrm{C} 9 \mathrm{H}) ; \delta(\mathrm{O} 3 \mathrm{H})\end{array}$ \\
\hline $1209(1211)$ & 1204 & $1205(1213)$ & $\delta_{i p}(\mathrm{C} 11 \mathrm{H}, \mathrm{C} 12 \mathrm{H}, \mathrm{C} 14 \mathrm{H}, \mathrm{C} 15 \mathrm{H}) ; \delta(\mathrm{O} 3 \mathrm{H})$ & $\begin{array}{l}\tau\left(\mathrm{C} 4 \mathrm{H}_{2}\right) ; \delta(\mathrm{C} 5 \mathrm{H}, \mathrm{C} 8 \mathrm{H}, \mathrm{C} 9 \mathrm{H}) ; \delta(\mathrm{O} 3 \mathrm{H}) ; \\
\mathrm{v}_{s} \quad(\mathrm{C} 9 \mathrm{C} 10, \mathrm{C} 11 \mathrm{C} 12, \mathrm{C} 14 \mathrm{C} 15) ; \\
\mathrm{v}_{s}(\mathrm{C} 16 \mathrm{~N} 1) ; \\
\delta_{i p}(\mathrm{C} 2 \mathrm{H}, \mathrm{C} 11 \mathrm{H}, \mathrm{C} 12 \mathrm{H}, \mathrm{C} 14 \mathrm{H}, \mathrm{C} 15 \mathrm{H})\end{array}$ \\
\hline $1223(1221)$ & & & $\tau\left(\mathrm{C} 4 \mathrm{H}_{2}, \mathrm{C}_{6} \mathrm{H}_{2}\right) ; \delta(\mathrm{C} 5 \mathrm{H}, \mathrm{C} 8 \mathrm{H}, \mathrm{C} 9 \mathrm{H})$ & $\begin{array}{l}\tau\left(\mathrm{C}_{4} \mathrm{H}_{2}, \mathrm{C} \mathrm{H}_{2}\right) ; \quad \delta(\mathrm{C} 5 \mathrm{H}, \mathrm{C} 8 \mathrm{H}, \mathrm{C} 9 \mathrm{H}) \\
v_{s}(\mathrm{C} 9 \mathrm{C} 10) ; \quad \delta_{i p}(\mathrm{C} 12 \mathrm{H}, \mathrm{C} 14 \mathrm{H}, \mathrm{C} 15 \mathrm{H}) \\
v_{s}(\mathrm{C} 16 \mathrm{~N} 1) ; \delta_{i p}(\mathrm{C} 1 \mathrm{H}, \mathrm{C} 2 \mathrm{H})\end{array}$ \\
\hline $1234(1229)$ & 1235 & 1236 & $\tau\left(\mathrm{C} 6 \mathrm{H}_{2}\right) ; \delta(\mathrm{C} 5 \mathrm{H}, \mathrm{C} 8 \mathrm{H}, \mathrm{C} 9 \mathrm{H})$ & $\tau\left(\mathrm{C} 2 \mathrm{H}_{2} ; \mathrm{C} \mathrm{H}_{2}\right) ; \delta(\mathrm{C} 5 \mathrm{H}, \mathrm{C} 8 \mathrm{H}, \mathrm{C} 9 \mathrm{H})$ \\
\hline $1241(1246)$ & 1256 & $(1245)$ & $\begin{array}{l}\tau\left(\mathrm{C} 4 \mathrm{H}_{2} ; \mathrm{C} \mathrm{H}_{2}\right) ; \\
\delta(\mathrm{O} 3 \mathrm{H})\end{array}$ & $\begin{array}{lr}\tau\left(\mathrm{C} \mathrm{H}_{2}\right) ; & \operatorname{wag}\left(\mathrm{C}_{4} \mathrm{H}_{2}\right) ; \\
\delta(\mathrm{C} 5 \mathrm{H}, \mathrm{C} 8 \mathrm{H}, \mathrm{C} 9 \mathrm{H}) ; & \delta(\mathrm{O} 3 \mathrm{H}) ; \\
\delta_{i p}(\mathrm{C} 1 \mathrm{H}, \mathrm{C} 2 \mathrm{H}) & \\
\end{array}$ \\
\hline $1247(1266)$ & 1251 & & $\begin{array}{l}\tau\left(\mathrm{C} 4 \mathrm{H}_{2} ; \mathrm{C}_{2} \mathrm{H}_{2}\right) ; \delta(\mathrm{C} 5 \mathrm{H}, \mathrm{C} 8 \mathrm{H}, \mathrm{C} 9 \mathrm{H}) \\
\delta(\mathrm{O} 3 \mathrm{H}) ; \delta_{i p}(\mathrm{C} 1 \mathrm{H}, \mathrm{C} 2 \mathrm{H})\end{array}$ & $\begin{array}{l}\tau\left(\mathrm{C} 4 \mathrm{H}_{2} ; \mathrm{C} 6 \mathrm{H}_{2}\right) ; \delta(\mathrm{C} 5 \mathrm{H}, \mathrm{C} 8) ; \\
\delta_{i p}(\mathrm{C} 1 \mathrm{H}, \mathrm{C} 2 \mathrm{H}) \\
\end{array}$ \\
\hline $1269(1287)$ & 1270 & $(1278)$ & $\tau\left(\mathrm{C} 4 \mathrm{H}_{2} ; \mathrm{C}^{2} \mathrm{H}_{2}\right) ; \delta(\mathrm{C} 8 \mathrm{H}) ; \delta_{i p}(\mathrm{C} 1 \mathrm{H}, \mathrm{C} 2 \mathrm{H})$ & $\begin{array}{l}\tau\left(\mathrm{C} 4 \mathrm{H}_{2} ; \mathrm{C} 6 \mathrm{H}_{2}\right) ; \delta(\mathrm{C} 5 \mathrm{H}, \mathrm{C} 8 \mathrm{H}, \mathrm{C} 9 \mathrm{H}) ; \\
\delta_{i p}(\mathrm{C} 1 \mathrm{H}, \mathrm{C} 2 \mathrm{H})\end{array}$ \\
\hline
\end{tabular}


TABLE I - continued from previous page

\begin{tabular}{|c|c|c|c|c|}
\hline$\omega_{\text {calc }}$ & $\omega_{F T-R a m a m}$ & $\omega_{F T-I R}$ & Vibrational Modes of Pilosine & Vibrational Modes of Epiisopilosine \\
\hline $1284(1306)$ & $1284(1279)$ & 1291 & $\begin{array}{l}\tau\left(\mathrm{C} 4 \mathrm{H}_{2} ; \mathrm{C}_{6} \mathrm{H}_{2}\right) ; \delta(\mathrm{C} 5 \mathrm{H}, \mathrm{C} 8 \mathrm{H}, \mathrm{C} 9 \mathrm{H}) \\
\delta(\mathrm{O} 3 \mathrm{H})\end{array}$ & $\begin{array}{l}\tau\left(\mathrm{C} \mathrm{H}_{2}\right) ; \delta(\mathrm{C} 5 \mathrm{H}, \mathrm{C} 8 \mathrm{H}, \mathrm{C} 9 \mathrm{H}) ; \quad \delta(\mathrm{O} 3 \mathrm{H}) ; \\
\delta(\mathrm{C} 2 \mathrm{H}), v_{s}(\mathrm{C} 2 \mathrm{~N} 2, \mathrm{C} 16 \mathrm{~N} 1) ; \operatorname{wag}\left(\mathrm{C}_{4} \mathrm{H}_{2}\right)\end{array}$ \\
\hline $1310(1309)$ & $1317(1313)$ & $(1317)$ & $\begin{array}{ll}\delta(\mathrm{C} 5 \mathrm{H}, \mathrm{C} 8 \mathrm{H}) ; & \delta(\mathrm{C} 2 \mathrm{H}), \\
v_{s}(\mathrm{C} 2 \mathrm{~N} 2, \mathrm{C} 16 \mathrm{~N} 1) & \end{array}$ & $\begin{array}{l}\delta(\mathrm{C} 5 \mathrm{H}, \mathrm{C} 8 \mathrm{H}, \mathrm{C} 9 \mathrm{H}) ; \delta(\mathrm{O} 3 \mathrm{H}) ; \delta(\mathrm{C} 2 \mathrm{H}), \\
v_{s}(\mathrm{C} 2 \mathrm{~N} 3, \mathrm{C} 16 \mathrm{~N} 1)\end{array}$ \\
\hline $1323(1321)$ & 1330 & $(1339)$ & $\tau\left(\mathrm{C}_{4} \mathrm{H}_{2} ; \mathrm{C}_{6} \mathrm{H}_{2}\right) ; \delta(\mathrm{C} 5 \mathrm{H}, \mathrm{C} 8 \mathrm{H})$ & $\begin{array}{l}\tau\left(\mathrm{C} 4 \mathrm{H}_{2} ; \mathrm{C} 6 \mathrm{H}_{2}\right) ; \delta(\mathrm{C} 2 \mathrm{H}, \mathrm{C} 4 \mathrm{H}, \mathrm{C} 5 \mathrm{H}) ; \\
\delta_{i p}(\mathrm{C} 11 \mathrm{H}, \mathrm{C} 12 \mathrm{H}, \mathrm{C} 14 \mathrm{H}, \mathrm{C} 15 \mathrm{H}) ; \delta(\mathrm{O} 3 \mathrm{H})\end{array}$ \\
\hline $1350(1345)$ & & & $\operatorname{wag}\left(\mathrm{C}_{4} \mathrm{H}_{2}\right) ; \delta(\mathrm{C} 5 \mathrm{H}, \mathrm{C} 8 \mathrm{H})$ & $\begin{array}{l}\text { wag }\left(\mathrm{C} 4 \mathrm{H}_{2} ; \mathrm{C} 6 \mathrm{H}_{2}\right) ; \delta(\mathrm{C} 1 \mathrm{H}, \mathrm{C} 2 \mathrm{H}, \mathrm{C} 5 \mathrm{H}, \mathrm{C} 8 \mathrm{H}) \\
\delta_{i p}(\mathrm{C} 11 \mathrm{H}, \mathrm{C} 12 \mathrm{H}, \mathrm{C} 14 \mathrm{H}, \mathrm{C} 15 \mathrm{H})\end{array}$ \\
\hline $1356(1360)$ & $1359(1353)$ & & $\begin{array}{l}\text { wag }\left(\mathrm{C} 4 \mathrm{H}_{2}\right) ; \delta(\mathrm{C} 1 \mathrm{H}, \mathrm{C} 2 \mathrm{H}, \mathrm{C} 5 \mathrm{H}, \mathrm{C} 8 \mathrm{H}) \\
\delta_{i p}(\mathrm{C} 11 \mathrm{H}, \mathrm{C} 12 \mathrm{H}, \mathrm{C} 14 \mathrm{H}, \mathrm{C} 15 \mathrm{H}) ; \delta(\mathrm{O} 3 \mathrm{H})\end{array}$ & $\begin{array}{l}\tau\left(\mathrm{C} 4 \mathrm{H}_{2} ; \mathrm{C} \mathrm{H}_{2}\right) ; \delta(\mathrm{C} 5 \mathrm{H}, \mathrm{C} 8 \mathrm{H}, \mathrm{C} 9 \mathrm{H}) \\
\delta(\mathrm{O} 3 \mathrm{H})\end{array}$ \\
\hline $1363(1363)$ & $(1362)$ & 1332 & $\delta_{i p}(\mathrm{C} 11 \mathrm{H}, \mathrm{C} 12 \mathrm{H}, \mathrm{C} 13 \mathrm{H}, \mathrm{C} 14 \mathrm{H}, \mathrm{C} 15 \mathrm{H})$ & $\delta_{i p}(\mathrm{C} 11 \mathrm{H}, \mathrm{C} 12 \mathrm{H}, \mathrm{C} 13 \mathrm{H}, \mathrm{C} 14 \mathrm{H}, \mathrm{C} 15 \mathrm{H})$ \\
\hline $1381(1379)$ & $1381(1379)$ & $(1383)$ & $\begin{array}{ll}\operatorname{wag}\left(\mathrm{C}_{6} \mathrm{H}_{2}\right) ; & \delta(\mathrm{C} 5 \mathrm{H}, \mathrm{C} 8 \mathrm{H}, \mathrm{C} 9 \mathrm{H}) ; \\
\mathrm{r}\left(\mathrm{C} 16 \mathrm{H}_{3}\right) & \\
\end{array}$ & $\begin{array}{l}\operatorname{wag}\left(\mathrm{C} 4 \mathrm{H}_{2}, \mathrm{C} 6 \mathrm{H}_{2}\right) ; \delta(\mathrm{C} 5 \mathrm{H}, \mathrm{C} 8 \mathrm{H}, \mathrm{C} 9 \mathrm{H}) ; \\
\mathrm{r}\left(\mathrm{C} 16 \mathrm{H}_{3}\right) ; v_{s}(\mathrm{C} 1 \mathrm{~N} 1)\end{array}$ \\
\hline $1387(1388)$ & & 1362 & wag $\left(\mathrm{C} \mathrm{H}_{2}\right) ; \delta(\mathrm{C} 8 \mathrm{H}, \mathrm{C} 9 \mathrm{H})$ & 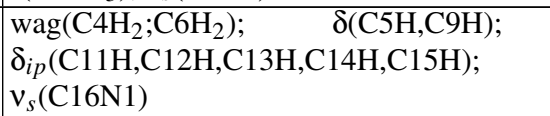 \\
\hline $1398(1392)$ & & & $\begin{array}{l}\operatorname{wag}\left(\mathrm{C}_{6} \mathrm{H}_{2}\right) ; \\
\delta(\mathrm{O} 3 \mathrm{H})\end{array}$ & $\begin{array}{l}\operatorname{wag}\left(\mathrm{C} 4 \mathrm{H}_{2} ; \mathrm{C} \mathrm{H}_{2}\right) ; \quad \delta(\mathrm{C} 5 \mathrm{H}, \mathrm{C} 9 \mathrm{H}) \\
\delta_{i p}(\mathrm{C} 11 \mathrm{H}, \mathrm{C} 12 \mathrm{H}, \mathrm{C} 13 \mathrm{H}, \mathrm{C} 14 \mathrm{H}, \mathrm{C} 15 \mathrm{H}) \\
\mathrm{v}_{S}(\mathrm{C} 16 \mathrm{~N} 1)\end{array}$ \\
\hline $1401(1413)$ & $(1424)$ & $(1387)$ & $\operatorname{wag}\left(\mathrm{C} 4 \mathrm{H}_{2}\right) ; \delta(\mathrm{C} 5 \mathrm{H}, \mathrm{C} 8 \mathrm{H}) ; v_{s}(\mathrm{C} 16 \mathrm{~N} 1)$ & $\begin{array}{l}\text { wag }\left(\mathrm{C} 4 \mathrm{H}_{2}, \mathrm{C} \mathrm{H}_{2}\right) ; \delta(\mathrm{C} 5 \mathrm{H}, \mathrm{C} 8 \mathrm{H}, \mathrm{C} 9 \mathrm{H}) ; \\
\delta(\mathrm{O} 3 \mathrm{H})\end{array}$ \\
\hline $1424(1420)$ & $1420(1437)$ & $1423(1436)$ & $\delta(\mathrm{C} 5 \mathrm{H}, \mathrm{C} 8 \mathrm{H}, \mathrm{C} 9 \mathrm{H}) ; \delta(\mathrm{O} 3 \mathrm{H}) ; v_{s}(\mathrm{C} 9 \mathrm{C} 10)$ & $\begin{array}{l}\text { wag }\left(\mathrm{C} 6 \mathrm{H}_{2}\right) ; \quad \delta(\mathrm{C} 5 \mathrm{H}, \mathrm{C} 8 \mathrm{H}, \mathrm{C} 9 \mathrm{H}) ; \\
\delta(\mathrm{O} 3 \mathrm{H}) ; v_{s}(\mathrm{C} 9 \mathrm{C} 10)\end{array}$ \\
\hline $1439(1439)$ & $1443(1452)$ & $1446(1452)$ & $\begin{array}{ll}\text { wag }\left(\mathrm{C} 4 \mathrm{H}_{2}, \mathrm{C} 16 \mathrm{H}_{3}\right) ; & \delta(\mathrm{C} 5 \mathrm{H}, \mathrm{C} 9 \mathrm{H}) ; \\
v_{S}(\mathrm{C} 1 \mathrm{~N} 1, \mathrm{C} 1 \mathrm{~N} 3, \mathrm{C} 3 \mathrm{~N} 1) & \\
\end{array}$ & $\begin{array}{ll}\text { wag }\left(\mathrm{C} 4 \mathrm{H}_{2}, \mathrm{C} 16 \mathrm{H}_{3}\right) ; & \delta(\mathrm{C} 5 \mathrm{H}) ; \\
\mathrm{v}_{S}(\mathrm{C} 1 \mathrm{~N} 3, \mathrm{C} 3 \mathrm{~N} 1) & \\
\end{array}$ \\
\hline $1469(1468)$ & 1454 (1469) & $1463(1470)$ & wag $\left(\mathrm{C} 16 \mathrm{H}_{3}\right)$ & $\operatorname{wag}\left(\mathrm{C}_{16 H_{3}}\right)$ \\
\hline $1496(1497)$ & 1465 & $(1494)$ & $\begin{array}{l}\mathrm{sc}\left(\mathrm{C} 4 \mathrm{H}_{2}, \mathrm{C} 16 \mathrm{H}_{3}\right) ; \\
\delta_{i p}(\mathrm{C} 11 \mathrm{H}, \mathrm{C} 12 \mathrm{H}, \mathrm{C} 13 \mathrm{H}, \mathrm{C} 14 \mathrm{H}, \mathrm{H}, \mathrm{C} 15 \mathrm{H})\end{array}$ & $\begin{array}{l}\mathrm{sc}\left(\mathrm{C} 4 \mathrm{H}_{2}, \mathrm{C} 16 \mathrm{H}_{3}\right) ; \quad \delta(\mathrm{C} 9 \mathrm{H}) ; \\
\delta_{i p}(\mathrm{C} 11 \mathrm{H}, \mathrm{C} 12 \mathrm{H}, \mathrm{C} 13 \mathrm{H}, \mathrm{C} 14 \mathrm{H}, \mathrm{C} 15 \mathrm{H})\end{array}$ \\
\hline $1497(1498)$ & 1480 & 1480 & $\begin{array}{l}\mathrm{sc}\left(\mathrm{C} 4 \mathrm{H}_{2}, \mathrm{C} 16 \mathrm{H}_{3}\right) ; \\
\delta_{i p}(\mathrm{C} 11 \mathrm{H}, \mathrm{C} 12 \mathrm{H}, \mathrm{C} 13 \mathrm{H}, \mathrm{C} 14 \mathrm{H}, \mathrm{C} 15 \mathrm{H})\end{array}$ & $\mathrm{sc}\left(\mathrm{C} 4 \mathrm{H}_{2}, \mathrm{C} 16 \mathrm{H}_{3}\right)$ \\
\hline $1503(1502)$ & $(1492)$ & 1495 & $\mathrm{sc}\left(\mathrm{C} 4 \mathrm{H}_{2}, \mathrm{C} 16 \mathrm{H}_{3}\right)$ & $\mathrm{sc}\left(\mathrm{C} 4 \mathrm{H}_{2}, \mathrm{C} \mathrm{H}_{2}, \mathrm{C} 16 \mathrm{H}_{3}\right)$ \\
\hline $1525(1526)$ & $1508(1513)$ & $1510(1513)$ & $\mathrm{sc}\left(\mathrm{C} 4 \mathrm{H}_{2}, \mathrm{C}_{16 \mathrm{H}_{3}}\right)$ & $\mathrm{sc}\left(\mathrm{C} 4 \mathrm{H}_{2}, \mathrm{C} \mathrm{H}_{2}, \mathrm{C}_{\left.16 \mathrm{H}_{3}\right)}\right.$ \\
\hline $1535(1532)$ & 1563 & 1563 & $\mathrm{sc}\left(\mathrm{C} 6 \mathrm{H}_{2}\right)$ & $\mathrm{sc}\left(\mathrm{C} 4 \mathrm{H}_{2}, \mathrm{C}_{6} \mathrm{H}_{2}, \mathrm{C} 16 \mathrm{H}_{3}\right)$ \\
\hline $1541(1538)$ & $(1564)$ & $(1558)$ & $\delta_{i p}(\mathrm{C} 11 \mathrm{H}, \mathrm{C} 12 \mathrm{H}, \mathrm{C} 14 \mathrm{H}, \mathrm{C} 15 \mathrm{H})$ & $\delta_{i p}(\mathrm{C} 11 \mathrm{H}, \mathrm{C} 12 \mathrm{H}, \mathrm{C} 14 \mathrm{H}, \mathrm{C} 15 \mathrm{H})$ \\
\hline $1543(1544)$ & $1582(1582)$ & 1583 & $\begin{array}{l}\text { wag }\left(\mathrm{C} 4 \mathrm{H}_{2}\right) ; \\
\mathrm{v}(\mathrm{C} 1 \mathrm{~N} 3)\end{array}$ & $\begin{array}{l}\operatorname{wag}\left(\mathrm{C} 4 \mathrm{H}_{2}\right) ; \\
v(\mathrm{Cc} 1 \mathrm{~N} 2, \mathrm{C} 16 \mathrm{H} 1) ; \mathrm{v}_{s}(\mathrm{C} 2 \mathrm{C} 3) ; \delta(\mathrm{C} 1 \mathrm{H})\end{array}$ \\
\hline $1601(1603)$ & $1603(1601)$ & 1601 & $\begin{array}{ll}v_{s}(\mathrm{C} 2 \mathrm{C} 3, \mathrm{C} 3 \mathrm{C} 4) ; & \delta_{i p}(\mathrm{C} 1 \mathrm{H}, \mathrm{C} 2 \mathrm{H}) \\
\mathrm{r}\left(\mathrm{C} 16 \mathrm{H}_{3}\right) & \end{array}$ & $\begin{array}{l}v_{s}(\mathrm{C} 2 \mathrm{C} 3, \mathrm{C} 3 \mathrm{C} 4) ; \\
\mathrm{r}\left(\mathrm{C} 16 \mathrm{H}_{3}\right)\end{array}$ \\
\hline $1644(1642)$ & & & $\begin{array}{l}\mathrm{v}_{s}(\mathrm{C} 10 \mathrm{C} 15, \mathrm{C} 10 \mathrm{C} 11, \mathrm{C} 12 \mathrm{C} 13, \mathrm{C} 13, \mathrm{C} 14) \\
\delta_{i p}(\mathrm{C} 11 \mathrm{H}, \mathrm{C} 12 \mathrm{H}, \mathrm{C} 13 \mathrm{H}, \mathrm{C} 14 \mathrm{H}, \mathrm{C} 15 \mathrm{H})\end{array}$ & $\begin{array}{l}v_{s}(\mathrm{C} 10 \mathrm{C} 15, \mathrm{C} 10 \mathrm{C} 11, \mathrm{C} 12 \mathrm{C} 13, \mathrm{C} 13 \mathrm{C} 14) \\
\delta_{i p}(\mathrm{C} 11 \mathrm{H}, \mathrm{C} 12 \mathrm{H}, \mathrm{C} 13 \mathrm{H}, \mathrm{C} 14 \mathrm{H}, \mathrm{C} 15 \mathrm{H}) \\
\delta(\mathrm{C} 9 \mathrm{H})\end{array}$ \\
\hline $1664(1662)$ & $(1725)$ & & $\begin{array}{l}v_{s}(\mathrm{C} 12 \mathrm{C} 13, \mathrm{C} 14 \mathrm{C} 15) \\
\delta_{i p}(\mathrm{C} 11 \mathrm{H}, \mathrm{C} 12 \mathrm{H}, \mathrm{C} 15 \mathrm{H}) \\
\end{array}$ & \begin{tabular}{|l|}
$v_{s}(\mathrm{C} 11 \mathrm{C} 12, \mathrm{C} 14 \mathrm{C} 15)$ \\
$\delta_{i p}(\mathrm{C} 11 \mathrm{H}, \mathrm{C} 12 \mathrm{H}, \mathrm{C} 14 \mathrm{H}, \mathrm{C} 15 \mathrm{H})$ \\
\end{tabular} \\
\hline $1877(1869)$ & $(1749)$ & $1760(1749)$ & $\mathrm{v}_{s}(\mathrm{C} 7 \mathrm{O} 2)$ & $\mathrm{v}_{s}(\mathrm{C} 7 \mathrm{O} 2)$ \\
\hline $3005(3001)$ & 2825 & $(2829)$ & $v_{s}\left(\mathrm{C} 4 \mathrm{H}_{2}\right) ; v(\mathrm{C} 8 \mathrm{H}, \mathrm{C} 9 \mathrm{H})$ & $\mathrm{v}_{s}\left(\mathrm{C} 4 \mathrm{H}_{2}\right) ; \mathrm{v}(\mathrm{C} 8 \mathrm{H}, \mathrm{C} 9 \mathrm{H})$ \\
\hline $3012(3003)$ & & $(2841)$ & $\mathrm{v}_{S}\left(\mathrm{C} 4 \mathrm{H}_{2}\right) ; \mathrm{v}(\mathrm{C} 8 \mathrm{H}, \mathrm{C} 9 \mathrm{H})$ & $\mathrm{v}_{s}\left(\mathrm{C} 4 \mathrm{H}_{2}\right) ; \mathrm{v}(\mathrm{C} 5 \mathrm{H}, \mathrm{C} 9 \mathrm{H})$ \\
\hline $3034(3029)$ & $(2963)$ & $(2857)$ & $\mathrm{v}(\mathrm{C} 5 \mathrm{H}, \mathrm{C} 8 \mathrm{H}, \mathrm{C} 9 \mathrm{H}) ; \mathrm{v}_{a s}\left(\mathrm{C} 4 \mathrm{H}_{2}\right)$ & $\mathrm{v}(\mathrm{C} 5 \mathrm{H}, \mathrm{C} 8 \mathrm{H}, \mathrm{C} 9 \mathrm{H})$ \\
\hline $3040(3045)$ & $2864(2885)$ & 2869 (2889) & $\begin{array}{ll}v_{s}\left(\mathrm{C}_{16 \mathrm{H}}\right) ; & \mathrm{v}_{a s}\left(\mathrm{C}_{4} \mathrm{H}_{2}, \mathrm{C} \mathrm{H}_{2}\right) ; \\
\mathrm{v}(\mathrm{C} 5 \mathrm{H}, \mathrm{C} 8 \mathrm{H}, \mathrm{C} 9 \mathrm{H}) & \end{array}$ & $\mathrm{v}_{a s}\left(\mathrm{C} 4 \mathrm{H}_{2}, \mathrm{C} \mathrm{H}_{2}\right) ; \mathrm{v}(\mathrm{C} 5 \mathrm{H}, \mathrm{C} 8 \mathrm{H})$ \\
\hline $3051(3049)$ & $2919(2908)$ & $(2907)$ & $v_{s}\left(\mathrm{C}_{16 \mathrm{H}_{3}}\right)$ & $\mathrm{v}_{s}\left(\mathrm{C} 16 \mathrm{H}_{3}\right)$ \\
\hline $3055(3064)$ & $2927(2926)$ & $(2926)$ & $v_{s}\left(\mathrm{C}_{6} \mathrm{H}_{2}\right) ; v_{a s}\left(\mathrm{C} 4 \mathrm{H}_{2}\right) ; v(\mathrm{C} 5 \mathrm{H})$ & $\mathrm{v}_{s}\left(\mathrm{C} 6 \mathrm{H}_{2}\right) ; \mathrm{v}_{a s}\left(\mathrm{C} 4 \mathrm{H}_{2}\right) ; \mathrm{v}(\mathrm{C} 5 \mathrm{H}, \mathrm{C} 8 \mathrm{H})$ \\
\hline $3098(3081)$ & $(2949)$ & $(2948)$ & $v_{s}\left(\mathrm{C} \mathrm{H}_{2}\right) ; \mathrm{v}_{a s}\left(\mathrm{C} 4 \mathrm{H}_{2}\right) ; v(\mathrm{C} 5 \mathrm{H})$ & $\mathrm{v}_{s}\left(\mathrm{C} \mathrm{H}_{2}\right) ; \mathrm{v}_{a s}\left(\mathrm{C} 4 \mathrm{H}_{2}\right) ; \mathrm{v}(\mathrm{C} 5 \mathrm{H})$ \\
\hline $3114(3111)$ & 2954 (2957) & $(2953)$ & $\mathrm{v}_{a s}\left(\mathrm{C} 16 \mathrm{H}_{3}\right)$ & $\mathrm{v}_{a s}\left(\mathrm{C}_{16 \mathrm{H}_{3}}\right)$ \\
\hline $3143(3146)$ & $2979(2966)$ & $(2969)$ & $\mathrm{v}_{a s}\left(\mathrm{C}_{6} \mathrm{H}_{2}\right)$ & $\mathrm{v}_{a s}\left(\mathrm{C}_{6} \mathrm{H}_{2}\right)$ \\
\hline $3153(3151)$ & (2993) & & $\mathrm{v}_{a s}\left(\mathrm{C}_{\left.16 \mathrm{H}_{3}\right)}\right.$ & $\mathrm{v}_{a s}\left(\mathrm{C}_{\left.16 \mathrm{H}_{3}\right)}\right)$ \\
\hline $3178(3178)$ & $2998(3005)$ & $3111(3003)$ & $\mathrm{v}_{a s}(\mathrm{C} 11 \mathrm{H}, \mathrm{C} 12 \mathrm{H}, \mathrm{C} 13 \mathrm{H}, \mathrm{C} 14 \mathrm{H}, \mathrm{C} 15 \mathrm{H})$ & $\mathrm{V}_{a s}(\mathrm{C} 11 \mathrm{H}, \mathrm{C} 12 \mathrm{H}, \mathrm{C} 13 \mathrm{H}, \mathrm{C} 14 \mathrm{H}, \mathrm{C} 15 \mathrm{H})$ \\
\hline $3188(3188)$ & $3031(3034)$ & (3023) & $\mathrm{v}_{a s}(\mathrm{C} 11 \mathrm{H}, \mathrm{C} 12 \mathrm{H}, \mathrm{C} 13 \mathrm{H}, \mathrm{C} 14 \mathrm{H}, \mathrm{C} 15 \mathrm{H})$ & $\mathrm{v}_{a s}(\mathrm{C} 11 \mathrm{H}, \mathrm{C} 12 \mathrm{H}, \mathrm{C} 13 \mathrm{H}, \mathrm{C} 14 \mathrm{H}, \mathrm{C} 15 \mathrm{H})$ \\
\hline $3199(3197)$ & $3055(3043)$ & (3044) & $\mathrm{V}_{a s}(\mathrm{C} 11 \mathrm{H}, \mathrm{C} 12 \mathrm{H}, \mathrm{C} 13 \mathrm{H}, \mathrm{C} 14 \mathrm{H}, \mathrm{C} 15 \mathrm{H})$ & $\mathrm{V}_{a s}(\mathrm{C} 11 \mathrm{H}, \mathrm{C} 12 \mathrm{H}, \mathrm{C} 13 \mathrm{H}, \mathrm{C} 14 \mathrm{H}, \mathrm{C} 15 \mathrm{H})$ \\
\hline $3209(3205)$ & $3063(3075)$ & & $\mathrm{v}_{a s}(\mathrm{C} 11 \mathrm{H}, \mathrm{C} 12 \mathrm{H}, \mathrm{C} 13 \mathrm{H}, \mathrm{C} 14 \mathrm{H}, \mathrm{C} 15 \mathrm{H})$ & $\mathrm{V}_{a s}(\mathrm{C} 11 \mathrm{H}, \mathrm{C} 12 \mathrm{H}, \mathrm{C} 13 \mathrm{H}, \mathrm{C} 14 \mathrm{H}, \mathrm{C} 15 \mathrm{H})$ \\
\hline $3212(3215)$ & $3112(3090)$ & $(3087)$ & $\mathrm{v}_{S}(\mathrm{C} 11 \mathrm{H}, \mathrm{C} 12 \mathrm{H}, \mathrm{C} 13 \mathrm{H}, \mathrm{C} 14 \mathrm{H}, \mathrm{C} 15 \mathrm{H})$ & $\mathrm{V}_{s}(\mathrm{C} 11 \mathrm{H}, \mathrm{C} 12 \mathrm{H}, \mathrm{C} 13 \mathrm{H}, \mathrm{C} 14 \mathrm{H}, \mathrm{C} 15 \mathrm{H})$ \\
\hline $3249(3247)$ & $3136(3126)$ & (3124) & $\mathrm{v}_{a s}(\mathrm{C} 1 \mathrm{H}, \mathrm{C} 2 \mathrm{H})$ & $\mathrm{v}_{a s}(\mathrm{C} 1 \mathrm{H}, \mathrm{C} 2 \mathrm{H})$ \\
\hline $3052(3052)$ & 3166 & & $\mathrm{v}_{s}(\mathrm{C} 1 \mathrm{H}, \mathrm{C} 4 \mathrm{H})$ & $\mathrm{V}_{S}(\mathrm{C} 1 \mathrm{H}, \mathrm{C} 4 \mathrm{H})$ \\
\hline
\end{tabular}


TABLE I - continued from previous page

\begin{tabular}{|c|l|l|l|l|}
\hline$\omega_{\text {calc }}$ & $\omega_{F T-R a m a m}$ & $\omega_{F T-I R}$ & Vibrational Modes of Pilosine & Vibrational Modes of Epiisopilosine \\
\hline $3826(3808)$ & & & $v(\mathrm{O} 3 \mathrm{H})$ & $v(\mathrm{O} 3 \mathrm{H})$ \\
\hline & \multicolumn{3}{|l}{} \\
\hline
\end{tabular}

The Figure 5 shows the atomic displacements corresponding to selected normal modes from the isolated molecular structure of pilosine and epiisopilosine.The calculated wavenumbers of pilosine molecule $\omega_{\text {calc }}=1019 \mathrm{~cm}^{-1}$ corresponds to the atomic displacements associated to deformations $\delta_{i p}(\mathrm{R} 1)$ and the calculed frequency $\omega_{\text {calc }}=1468$ $\mathrm{cm}^{-1}$ observed in epiisopilosine molecule corresponds to the atomic displacements associated to deformations of wag $\left(\mathrm{C}_{16} \mathrm{H}_{3}\right)$. These deformations correspond, respectively, to the strong Raman bands observed at $1031 \mathrm{~cm}^{-1}$ and to the medium Raman bands observed at $1469 \mathrm{~cm}^{-1}$.

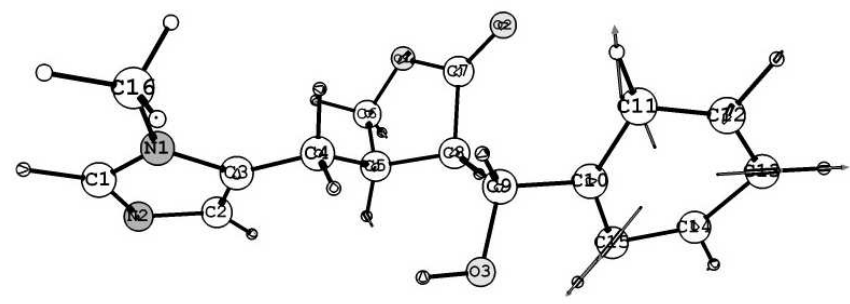

(a) $\omega_{\text {calc }}=1019 \mathrm{~cm}^{-1}$

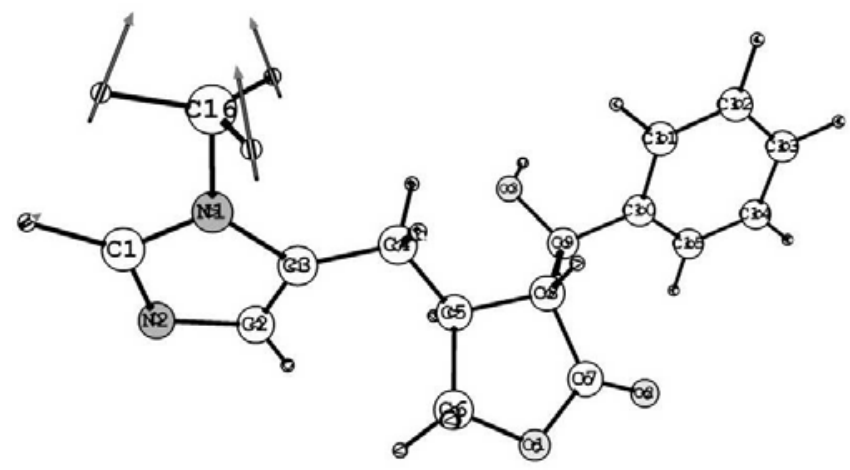

(b) $\omega_{\text {calc }}=1468 \mathrm{~cm}^{-1}$

FIG. 5: Selected representations of atomic vibrations corresponding to the calculated wavenumbers of molecules (a) pilosine and (b) epiisopilosine.

In the table I it is observed that some experimental frequencies $\omega_{F T-R a m a n}$ and $\omega_{F T-I R}$ are present in only one of the isomers. As an example, the experimental frequencies corresponding to the Raman bands $\left(\omega_{F T-\text { Raman }}\right)$ at $204 \mathrm{~cm}^{-1}$, $413 \mathrm{~cm}^{-1}$ and $474 \mathrm{~cm}^{-1}$ are present only in pilosine isomer. There are no Raman bands associated with these frequencies in the epiisopilosine isomer. In the same way, experimental frequencies of IR bands $\left(\omega_{F T-I R}\right)$ corresponding to 666 $\mathrm{cm}^{-1}, 966 \mathrm{~cm}^{-1}, 976 \mathrm{~cm}^{-1}$, e $1020 \mathrm{~cm}^{-1}$ are present only in epiisopilosine isomer. There are no IR bands associated with these frequencies for the pilosine isomer.
It is possible to note a marked localization of the wagging vibrations in the range $1350 \mathrm{~cm}^{-1}<\omega_{\text {calc }}<1459 \mathrm{~cm}^{-1}$. As an example, the Raman band observed at $1454 \mathrm{~cm}^{-1}\left(\omega_{\text {calc }}=\right.$ $\left.1469 \mathrm{~cm}^{-1}\right)$ corresponds to the vibration $\operatorname{wag}\left(\mathrm{C}_{16} \mathrm{H}_{3}\right)$ in pilosine molecule. This mode is also observed in epiisopilosine isomer.

Vibration type scissoring are located in the region 1496 $\mathrm{cm}^{-1}<\omega_{\text {calc }}<1543 \mathrm{~cm}^{-1}$. For example, the IR band observed at $1510 \mathrm{~cm}^{-1}\left(\omega_{\text {calc }}=1525 \mathrm{~cm}^{-1}\right)$ corresponds to the vibration $\mathrm{sc}\left(\mathrm{C} 4 \mathrm{H}_{2}, \mathrm{C}_{\left.16 \mathrm{H}_{3}\right)}\right.$ in the pilosine molecule. In epiisopilosine molecule the Raman and IR bands are located at $1513 \mathrm{~cm}^{-1}\left(\omega_{\text {calc }}=1526 \mathrm{~cm}^{-1}\right)$ and corresponds to the atomic displacements associated to deformations $\mathrm{sc}\left(\mathrm{C} 4 \mathrm{H}_{2}, \mathrm{C}_{6} \mathrm{H}_{2}, \mathrm{C} 16 \mathrm{H}_{3}\right)$.

Another class of vibration is related to deformation of rings. In plane ring deformation vibration appears in a large spectral region $\left(898 \mathrm{~cm}^{-1}<\omega_{\text {calc }}<1664 \mathrm{~cm}-1\right)$ and out of plane ring deformation vibration appears for $279 \mathrm{~cm}^{-1}<$ $\omega_{\text {calc }}<997 \mathrm{~cm}^{-1}$.

The spectral region between $2800 \mathrm{~cm}^{-1}$ and $3200 \mathrm{~cm}^{-1}$ of the Raman spectrum of pilosine crystal consists of a series of very intense Raman bands, and a series of less intense IR bands. However, all bands are well resolved, allowing for their identification as listed in Table I.

For organic crystals the region about $3000 \mathrm{~cm}^{-1}$, in general, contains the bands originated from $\mathrm{CH}, \mathrm{CH}_{2}, \mathrm{CH}_{3}$, and $\mathrm{NH}$ vibrations $[17,18]$. For some materials this region condenses very important informations, being a tool to understand conformation of the molecules in the unit cell or even interactions such hydrogen bonds. For example, a study on Lmethionine crystal have shown that the behaviour of Raman bands under pressure in this spectral region can be understood as consequence of structural changes instead of simple conformational changes of molecules in the unit cell [19]. So, the understand of the origin of these bands can be fundamental to understand the behaviour of pilosine under different conditions, in particular, related to the conditions found in drug artefacts. Our work (see Table I) gives a very precise assignment for bands appearing in this region for both pilosine and epiisopilosine isomers.

\section{CONCLUSIONS}

The phonon spectra of the pilosine and epiisopilosine crystals, potential pharmaceutical substances to be used in several disease treatments, were measured at room temperature through FT-Raman and FT-IR techniques. Density functional theory calculations were carried out by using the Gaussian 98 package and the B3LYP functional with the 6-31 G(d,p) basis set. The calculations were observed to reproduce the experiments with good agreement. This agreement allowed 
us the assignment of the observed wavenumbers to atomic motions in the molecules. In particular, it was observed that most bands are associated to mixing of vibrational modes, even in the low wavenumber region where, generally, the lattice modes are found. The absence of stretching vibrations of water molecule at $3400 \mathrm{~cm}^{-1}$ in this region indicates that the crystal is free of water molecules.

\section{Acknowledgements}

The authors would like to acknowledge the financial support of the Brazilian agencies FUNCAP and CNPq and the
CENAPAD-SP for the use of the GAUSSIAN 98 software package and for computational facilities through the project reference proj373.
[1] C.U.B. Pinheiro, Econ. Bot. 51, 49 (1997).

[2] C. Migdal, Eye 14, 515 (2000).

[3] R.L. Wynn, Gen. Dent. 44, 29 (1996)

[4] A.N. Davies, K. Broadley, D. Beighton, J. Pain Sympt. Manag. 22, 820 (2001).

[5] I.N. Abreu, A.C.H.F. Sawaya, M.N. Eberlin, P. Mazzafera, In vitro Cell Dev. Biol. Plant.41, 806 (2005).

[6] J. Pelletier and Magendie, Ann. Chim.phys., (2), 4, 172 (1817).

[7] A. Glnard, Compt.rend., 81, 100 (1875).

[8] M.J. Frisch, G.W. Trucks, H.B. Schlegel, G.E. Scuseria, M.A. Robb, J.R. Cheeseman, V.G. Zakrzewski, J.A. Montgomery, R.E. Stratmann Jr, J.C. Burant, S. Dapprich, J. M. Millam, A.D. Daniels, K.N. Kudin, M.C. Strain, O. Farkas, J. Tomasi, V. Barone, M. Cossi, R. Cammi, B. Mennucci, C. Pomelli, C. Adamo, S. Clifford, J. Ochterski, G.A. Petersson, P.Y. Ayala, Q. Cui, K. Morokuma, P. Salvador, J.J. Dannenberg, D.K. Malick, A.D. Rabuck, K. Raghavachari, J.B. Foresman, J. Cioslowski, J.V. Ortiz, A.G. Baboul, B.B. Stefanov, G. Liu, A. Liashenko, P. Piskorz, I. Komaromi, R. Gomperts, R.L. Martin, D.J. Fox, T. Keith, M.A. Al-Laham, C.Y. Peng, A. Nanayakkara, M. Challacombe, P.M.W. Gill, B. Johnson, W. Chen, M.W. Wong, J.L. Andres, C. Gonzalez, M. HeadGordon, E.S. Replogle, J.A. Pople. Gaussian 98 (Revision A.11.2). Gaussian: Pittsburgh, PA, 2001.

[9] M. Tasumi , I. Harada, T. Takamatsu, S. Takahashi, J. Raman
Spectrosc. 12, 149 (1982)

[10] T. Miura, T. Satoh, A. Hori-i, H.J. Takeuchi, J. Raman Spectrosc. 29, 41 (1998);

[11] J.L.B. Faria, F.M. Almeida, O. Pilla, F. Rossi, J.M. Sasaki, F.E.A. Melo, J.M. Filho, P.T.C. Freire, J. Raman Spectrosc. 35, 242 (2004).

[12] A. Torreggiani, A. Degli Esposti, M. Tamba, G. Marconi, G. Fini, J. Raman Spectrosc. 37, 291 (2006).

[13] B.H. Loo, Y. Tse, K. Parsons, C. Adelman, A. El-Hage, Y.G. Lee, J. Raman Spectrosc. 37, 299 (2006).

[14] W. Zhang, K. Krohn, J. Ding, Z.H. Miao, X. H. Zhou, S.H. Chen, G. Pescitelli, P. Salvadori, T. Kurtan, Y.W. Guo, J. Nat. Prod. 71, 961 (2008).

[15] J. Binoy, J. P. Abraham, I. Hybert Joe, V. George, V.S. Jayakumar, J. Aubard, O. Faurskov Nielsen, J. Raman Spectrosc. 36, 63 (2005).

[16] S. Basu, Y. Gerchman, C.H. Collins, F.H. Arnold, R. Weiss, Nature 434, 1130 (2005)

[17] B.L.Silva, P.T.C. Freire, F.E.A. Melo, I. Guedes, M.A.A. Silva, J. M. Filho, A.J.D. Moreno, Braz. J. Phys. 2819 (1998).

[18] P. F. Faanha Filho, P.T.C. Freire, K.C.V. Lima, J.M. Filho, F.E.A. Melo, P.S. Pizani, Braz. J. Phys. 38, 131 (2008).

[19] J.A. Lima Jr., P.T.C. Freire, F.E.A. Melo, V. Lemos, J. MendesFilho, P.S. Pizani, J. Raman Spectrosc. 39, 1356 (2008). 